\title{
Critical appraisal of a fixed combination of esomeprazole and low dose aspirin in risk reduction
}

This article was published in the following Dove Press journal:

Therapeutics and Clinical Risk Management

19 June 2010

Number of times this article has been viewed

\author{
Ravi Vachhani' \\ Doumit Bouhaidar' \\ Alvin Zfass' \\ Bimaljit Sandhu' \\ Ali Nawras ${ }^{2}$ \\ 'Division of Gastroenterology, \\ Hepatology and Nutrition, \\ Department of Internal Medicine, \\ Virginia Commonwealth University \\ Medical Center, Richmond,Virginia \\ 23298-034I, USA; ${ }^{2}$ Division of \\ Gastroenterology, Hepatology and \\ Nutrition, Department of Internal \\ Medicine, The University of Toledo \\ Medical Center, Toledo, Ohio \\ 43606-3390, USA
}

\begin{abstract}
Low dose aspirin $(\leq 325 \mathrm{mg})$ is routinely used for primary and secondary prophylaxis of cardiovascular and cerebrovascular events. The use of low dose aspirin is associated with twoto four-fold greater risk of symptomatic or complicated peptic ulcers. Risk factors associated with low dose aspirin induced gastrointestinal toxicity includes prior history of ulcer or upper gastrointestinal (GI) bleeding, concomitant use of other nonsteroidal anti-inflammatory drugs, corticosteroid or warfarin, dual antiplatelet therapy, Helicobacter pylori (H. pylori) infection, and advanced age. Esomeprazole, like other proton pump inhibitors (PPIs) is very effective in decreasing the risk of aspirin induced gastrointestinal toxicity. Although evidence to support esomeprazole or other PPIs for primary prophylaxis in aspirin induced gastrointestinal toxicity is limited, its role in secondary prophylaxis is well established.
\end{abstract}

Keywords: esomeprazole, proton pump inhibitors, low dose aspirin, gastrointestinal toxicity, gastrointestinal bleeding

Low dose aspirin is routinely used for primary and secondary prophylaxis of cardiovascular and cerebrovascular events. ${ }^{1,2}$ Low dose aspirin is commonly defined as $\leq 325 \mathrm{mg}$ daily as compared to dose $>325 \mathrm{mg}$ and up to 4 gm daily used for antiinflammatory and analgesic properties. ${ }^{3,4}$ It has been estimated that during the past two decades 50 million Americans have started taking aspirin for the prevention of heart attack and stroke. ${ }^{5}$

Like other nonsteroidal anti-inflammatory drugs (NSAIDs), aspirin use can be limited by its association with mucosal injury to the upper GI tract, including the development of peptic ulcer disease (PUD) and complicating hemorrhage, perforation and death. ${ }^{6-8}$

\section{Mechanism of antithrombotic action and GI toxicity}

The primary effect of aspirin, like other NSAIDs, is to inhibit cyclooxygenase (COX) (prostaglandin synthase), thereby impairing the ultimate transformation of arachidonic acid to prostaglandins (PGs), prostacyclin, and thromboxanes. ${ }^{9}$ Two isoforms of the COX enzyme have been described: COX-1 and COX-2.

COX-1 (constitutional) is variably expressed in most tissues. It regulates normal cellular processes such as gastric cytoprotection and platelet aggregation. COX-2 (inducible) is usually undetectable in most tissues; its expression is increased during states of inflammation.
Correspondence: Ravi Vachhani Virginia, Commonwealth University Medical Center, Richmond,Virginia 23298-034I, USA

$\mathrm{Tel}+\mathrm{I}(804)-828-4060$

$\mathrm{Fax}+\mathrm{I}(804)-828-5348$

Email rvachhani@mcrh-vcu.edu 
Aspirin produces its antithrombotic effect by inhibiting the production of thromboxane A2 secondary to inhibition of COX-1.

The healthy gastric and duodenal mucosae constitutively use COX-1 to produce its mucosal protective PGs. Some of the cytoprotective mechanisms of PGs include: stimulation of glycoprotein (mucin), bicarbonate, and phospholipid secretion by cells, enhancement of mucosal blood flow and oxygen delivery to cells via local vasodilation, increased epithelial cell migration and enhanced epithelial cell proliferation. ${ }^{10} \mathrm{COX}-1$ inhibition by aspirin leading to prostaglandin depletion impairs these protective factors, resulting in a gastric environment that is more susceptible to topical attack by endogenous factors, such as acid, pepsin, and bile salts. The human stomach requires five to eight days to recover its COX-1 activity and its ability to synthesize protective PGs, suggesting a very slow turnover of gastric COX-1. ${ }^{11}$

\section{Risk of GI toxicity with low dose aspirin}

In a meta-analysis of 14 trials including over 57,000 patients, relative risk (RR) of major GI bleeding with the use of low dose aspirin as compared with placebo was 2.07 (95\% confidence interval (CI): 1.61-2.66). The absolute rate increase with aspirin above placebo was $0.12 \%$ per year. ${ }^{12}$ In another meta-analysis of 24 trials including low dose and high dose aspirin, the odds ratio (OR) for bleeding with aspirin was $1.68 \%$ (95\% CI: $1.51-1.88){ }^{5}$

It is interesting to note that patients using low dose aspirin are usually "high risk" cohorts for GI events. A large 5 -year observational cohort study showed $0.23 \%$ background incidence of upper GI bleeding hospitalization in the group matched to the low dose aspirin users - nearly four times the rate noted for the general population without medications or condition predisposing to bleeding - suggesting that the group taking low dose aspirin was a "higher risk" population. ${ }^{13}$

\section{Risk factor for aspirin induced GI toxicity}

In a large Danish cohort, factors that increased the risk of gastrointestinal bleeding included prior history of ulcers or gastrointestinal bleeding, corticosteroid use, anticoagulant therapy and addition of a nonaspirin nonsteroidal antiinflammatory drug. ${ }^{13}$

\section{Prior history of ulcer or upper Gl bleeding}

A case control study of patients hospitalized with upper GI bleeding identified a prior history of upper GI bleeding [OR: 6.5 (95\% CI: 2.0-21.2)] and a prior history of ulcer [OR: 2.0 (95\% CI: 1.0-4.1)] as significant risk factors for hospitalization with upper GI bleeding in patients taking low dose aspirin. ${ }^{14} \mathrm{An}$ assessment of 903 patients discharged from a cardiology service on 75-325 mg of aspirin daily and followed for a mean of 45 months noted a significant association between hospitalization for upper GI bleeding and a history of peptic ulcer or upper GI bleeding (risk ratio: 3.1, $95 \%$ CI: $1.5-6.5) .{ }^{15}$

\section{Concomitant use of NSAID}

Addition of nonaspirin NSAIDs to low dose aspirin increases the risk of upper GI bleeding by two- to fourfold.

A case control study for hospitalization for bleeding ulcer revealed that low dose aspirin alone was associated with an adjusted OR of 3.3 (2.5-4.4), while the combination of low dose aspirin plus an NSAID had an OR of 7.7 $(3.6-16.4) .{ }^{16}$

Annual incidence of hospital admission for upper GI bleeding was more than doubled when NSAIDs plus low dose aspirin were compared with low dose aspirin alone: $1.4 \%$ vs $0.6 \%$. The relative risk for aspirin alone was 2.6 (2.2-2.9), and it was significantly higher: 5.6 (4.4-7) for low dose aspirin plus NSAID. ${ }^{13}$

Another similar study showed OR of 3.8 (1.8-7.8) for increased risk of upper GI bleeding with NSAID plus low dose aspirin compared to low dose aspirin alone. ${ }^{14}$

\section{Helicobacter pylori infection}

The interaction between $H$. pylori infection and NSAIDs in the pathogenesis of PUD remains controversial. A metaanalysis, which included data from 25 observational studies, demonstrated that both H. pylori infection and nonselective NSAID use are independent risk factors, these risk factors are at least additive and possibly synergistic for the development of peptic ulcer and ulcer bleeding. ${ }^{17}$

Chan et al studied whether eradication of the H. pylori infection alone is effective in preventing recurrent bleeding. Two hundred and fifty patients taking aspirin who presented with upper gastrointestinal bleeding (which was confirmed by endoscopy) were then randomly assigned separately to receive $20 \mathrm{mg}$ of omeprazole daily for six months or one week of eradication therapy followed by placebo for six months. 
Prior to randomization, their ulcers were healed by daily treatment with $20 \mathrm{mg}$ of omeprazole for eight weeks or longer. The probability of recurrent bleeding during the six-month period was $1.9 \%$ for patients who received eradication therapy and $0.9 \%$ for patients who received omeprazole (absolute difference, $1.0 \%$; 95\% CI: -1.9 to $3.9 \%$ ).

This study showed that among patients with $H$. pylori infection and a history of upper gastrointestinal bleeding who are taking low dose aspirin, the eradication of $H$. pylori is equivalent to treatment with omeprazole in preventing recurrent bleeding. ${ }^{18}$

In another randomized trial, all aspirin users with $H$. pylori infection and a history of ulcer bleeding received a course of eradication therapy. They were then randomly assigned to receive lansoprazole $(n=62)$ or placebo $(\mathrm{n}=61)$ for up to 12 months. It was found that $1.6 \%(95 \%$ CI: 0-9\%) of patients in the lansoprazole group compared with $14.8 \%$ (95\% CI: 7-26\%) in the placebo group had recurrent ulcer bleeding. In the latter study, however, two-thirds of the patients with recurrent ulcer bleeding in the placebo group either had failure of $H$. pylori eradication or used concomitant NSAIDs, making data interpretation very difficult. ${ }^{19}$

In a prospective cohort study, the incidence rates of ulcer bleeding were compared among three different cohorts of low dose aspirin users, namely: patients without prior ulcer history who just started using aspirin $(n=548)$; aspirin users with prior ulcer bleeding and $H$. pylori infection who had successful eradication of $H$. pylori $(\mathrm{n}=250)$; and $H$. pylorinegative aspirin users who had prior ulcer bleeding $(n=118)$. All patients received low dose aspirin ( $<160 \mathrm{mg}$ daily) without a gastroprotective agent. After a median followup of 48 months, the annualized incidence rate of ulcer bleeding in the three groups was $0.5 \%, 1.1 \%$, and $4.6 \%$, respectively. This study showed that confirmed eradication of $H$. pylori in aspirin users with prior ulcer bleeding significantly and substantially reduces the risk of recurrent bleeding. ${ }^{20}$

Two hundred and forty-five symptomatic elderly who were taking aspirin 75-300 mg daily, at least during the last 3 months, were evaluated by endoscopy. One hundred and twelve patients were $H$. pylori-positive and 133 patients were $H$. pylori-negative. A significantly higher prevalence of peptic ulcers was observed in $H$. pylori-positive than in $H$. pylori-negative subjects $(36.6 \%$ versus $15.8 \%$, $P=0.0002$ ). This study showed that $H$. pylori infection influences the prevalence of peptic ulcers and the cost effectiveness of the PPI prevention therapy. ${ }^{21}$

\section{Concomitant use of clopidogrel}

Addition of clopidogrel to aspirin increases the risk of GI and non GI bleeding. In the 'clopidogrel in unstable angina to prevent recurrent events' (CURE) trial, major bleeding (GI and non GI causes of bleeding) was significantly more common in the clopidogrel plus aspirin group (3.7\%) as compared with $2.7 \%$ in the aspirin plus placebo group; RR, 1.38; $95 \%$ CI: 1.13 to $1.67 ; P=0.001){ }^{22}$

In the 'management of atherothrombosis with clopidogrel in high-risk patients with recent transient ischemic attacks or ischemic stroke' (MATCH) trial, life threatening bleeding was higher in the group receiving aspirin and clopidogrel versus clopidogrel alone (96 [2.6\%] vs 49 [1.3\%]; absolute risk increase $1.3 \%$ [95\% CI: 0.6 to 1.9$]$ ]). The majority of bleeding was due to GI related complications. ${ }^{23}$

In the 'clopidogrel for high atherothrombotic risk and ischemic stabilization, management, and avoidance' (CHARISMA) trial, the rate of moderate bleeding was $2.1 \%$ in the clopidogrel plus aspirin group, as compared with $1.3 \%$ in the placebo plus aspirin group (RR, 1.62; 95\% CI: 1.27 to $2.08 ; P<0.001){ }^{24}$

These trials provide the evidence that combined low dose aspirin and clopidogrel therapy is associated with significantly higher risk of GI bleeding when compared with low dose aspirin alone.

\section{Age}

Though data regarding high risk of GI complication with low dose aspirin use in older population is mixed, ${ }^{15,25}$ Patrono et al showed that the risk was below $0.5 \%$ for patients under 50 years old, while the risk was $4 \%$ in controls aged 70-79 years old, and approximately $6 \%$ in controls over 80 years old. ${ }^{26}$

\section{Warfarin}

In a comprehensive meta-analysis of 14 randomized controlled trials (RCT) including 25,307 patients, results showed OR of 2.2 (95\% CI: 1.64-2.96) for major extracranial bleeding when aspirin alone was compared to combination of aspirin with warfarin. ${ }^{27}$

\section{Corticosteroid}

A cohort study showed that relative risk of hospitalization for upper GI bleeding was 5.3 (2.9-8.8) when low dose aspirin was used along with corticosteroid, ${ }^{28}$ as compared to RR of 2.6 (2.2-2.9) expected for the same population with low dose aspirin alone. ${ }^{13}$ 


\section{Low versus high dose aspirin}

Increasing the dose of aspirin above the cardioprotective dose increases the risk of GI bleeding compared to low dose aspirin. ${ }^{29}$ However, increasing the dose from $75-81 \mathrm{mg}$ to $325 \mathrm{mg}$ doesn't increase the risk of GI bleeding. In a meta-analysis the relative risk of major GI bleeding with "lower" low dose aspirin (75-162.5 mg daily) was similar to the relative risk with "higher" low dose aspirin (>162.5-325 mg daily): 2.22 (95\% CI: 1.61-3.06) vs 2.35 (95\% CI: 0.98-5.66). ${ }^{12}$ Two other randomized controlled trials have reported similar findings. ${ }^{30,31}$

Enteric-coated aspirin has no significant protective effect on the clinically relevant end point of gastrointestinal bleeding or other ulcer complications. ${ }^{32}$

\section{Mucosal protection}

Methods commonly employed to prevent the development of peptic ulceration and mucosal injury in patients taking aspirin include cotherapy with a PPI, high dose histamine-2 receptor antagonists (H2RA), or the synthetic prostaglandin E1 analog, misoprostol.

\section{Misoprostol}

Limited number of studies address the role of misoprostol specifically in aspirin. ${ }^{33-36}$ Most of the studies have been in nonaspirin NSAIDs. A meta-analysis shows that cotherapy with misoprostol reduced the incidence of duodenal ulcers by $53 \%$ and gastric ulcer by $74 \%$ when compared with the placebo in patients with chronic NSAIDs use. ${ }^{37}$

The usefulness of misoprostol was limited by the occurrence of GI side effects, primarily cramping and diarrhea, and by compliance problems related to four-times a day (q.i.d). dosage.

\section{High dose H2RA}

Systemic reviews have shown that double dose but not single dose H2RA are effective in reducing the risk of NSAIDinduced endoscopic gastric ulcers. ${ }^{37,38}$

\section{Proton pump inhibitors}

A number of studies have demonstrated that the PPIs are very effective for the healing and prevention of gastroduodenal ulcers associated with NSAIDs (including aspirin) when NSAIDs cannot be discontinued. ${ }^{18,19,39-43}$

Three trials specifically looked at the role of esomeprazole with low dose aspirin in prevention of gastroduodenal complications.

The role of aspirin with esomeprazole versus clopidogrel to prevent recurrent ulcer bleeding was assessed by Chan et $\mathrm{al}^{44}$ In a 12 -month, prospective, randomized, double-blind trial that compared aspirin plus esomeprazole with clopidogrel for patients who had previous aspirin-induced ulcer bleeding and were expected to continue low dose aspirin. Before randomization, healing of ulcers and eradication of H. pylori, if present, was documented. One hundred and fifty patients were assigned to receive aspirin plus esomeprazole and 161 patients were assigned to receive clopidogrel. The primary end point was recurrent ulcer bleeding as defined according to prespecified criteria - namely, hematemesis or melena with ulcers or bleeding erosions confirmed on endoscopy, or a decrease in the hemoglobin level of at least 2 $\mathrm{g}$ per deciliter in the presence of endoscopically documented ulcers or bleeding erosions.

Recurrent ulcer bleeding occurred in 13 patients receiving clopidogrel and 1 receiving aspirin plus esomeprazole. The cumulative incidence of recurrent bleeding during the 12 month period was $8.6 \%$ (95\% CI: 4.1-13.1\%) among patients who received clopidogrel and $0.7 \%$ (95\% CI: $0-2.0 \%)$ among those who received aspirin plus esomeprazole (difference: $7.9 \%$ points; $95 \%$ CI: $3.4-12.4 ; P=0.001){ }^{44}$

In a similar trial, the role of esomeprazole with aspirin versus clopidogrel for prevention of recurrent gastrointestinal ulcer complications was assessed in a prospective, double-blind, randomized, controlled study of 170 patients. Patients who developed ulcer bleeding after the use of low dose and were expected to require continuous treatment with low dose aspirin were randomly assigned to treatment with esomeprazole $20 \mathrm{mg}$ /day and aspirin $100 \mathrm{mg}$ /day $(\mathrm{n}=86)$ or clopidogrel $75 \mathrm{mg} /$ day $(\mathrm{n}=84)$ for 52 weeks. Before randomization, healing of ulcers and eradication of H. pylori, if present, was documented. The primary end point was recurrent ulcer complications. During a median follow up period of 52 weeks, no patient in the esomeprazole group, as compared with 9 patients in the clopidogrel group, developed recurrent ulcer complications. The cumulative incidences of recurrent ulcer complications were $0 \%$ in patients receiving esomeprazole and aspirin and $13.6 \%$ in patients receiving clopidogrel (absolute difference, 13.6\%; 95\% CI: 6.3-20.9; $P=0.0019) \cdot{ }^{45}$

A multinational trial by Yeomans et al assessed the efficacy of esomeprazole $20 \mathrm{mg}$ once daily vs the placebo for primary risk reduction of the gastroduodenal ulcers associated with continuous use of low dose aspirin. In this randomized, double-blind, placebo controlled study patients aged $>60$ years old, who were receiving aspirin 75-325 mg once daily and without baseline gastroduodenal ulcer at endoscopy, were randomized to esomeprazole $20 \mathrm{mg}$ once daily or placebo for $26 \mathrm{wks}$. The presence of endoscopic 
gastric and/or duodenal ulcers and esophageal lesions was assessed at weeks 8 and 26. Upper gastrointestinal symptoms were assessed at weeks 8,16 , and 26 . The intention-to-treat population comprised 493 patients in the esomeprazole group and 498 patients in the placebo group. Twenty-seven patients $(5.4 \%)$ in the placebo group developed a gastric or duodenal ulcer during 26 weeks' treatment compared with eight patients $(1.6 \%)$ in the esomeprazole group (life table estimates: $6.2 \%$ vs $1.8 \% ; P=0.0007)$. At 26 weeks, the cumulative proportion of patients with erosive esophagitis was significantly lower for esomeprazole versus the placebo ( $4.4 \%$ and $18.3 \%$, respectively; $P<0.0001)$. At 26 weeks, esomeprazole treated patients were more likely to experience resolution of heartburn, acid regurgitation, and epigastric pain $(P<0.05){ }^{3}$

Pharmacokinetics and pharmacodynamics of esomeprazole are described elsewhere, ${ }^{46}$ there is no pharmacokinetic interaction between esomeprazole $(40 \mathrm{mg})$ and aspirin (325 mg) during repeated coadministration in healthy volunteers. ${ }^{47}$

\section{Discussion}

The role of PPI in prevention of low dose aspirin-induced gastrointestinal toxicity should be considered in the context of whether PPI is being considered for primary or secondary prevention of gastrointestinal toxicity.

Though the role of PPI for prevention of peptic ulcer in patients with chronic low dose aspirin is well documented, the evidence to support their use for prevention of a first episode of aspirin induced GI bleeding is limited.

In many studies, the endoscopic visualization of an ulcer was utilized and interpreted as a surrogate end point for GI bleeding and other complications of gastric and duodenal ulcers. However, the end point that really matters in clinical practice and against which the clinically relevant therapeutic effect of PPI must ultimately be judged is the prevention of GI complications and upper GI bleeding in particular. More randomized trials with clinical rather than endoscopic endpoints are needed before primary prevention with a PPI can be widely recommended. One can consider eradication of $H$. pylori, if positive, in a high-risk population prior to PPI use.

The role of PPI for secondary prevention of peptic ulcer in patients with chronic low dose aspirin is proven beyond doubt; however it is not unreasonable to consider eradication of $H$. pylori alone without PPI prophylaxis in chronic low dose aspirin users.

It has been suggested that gastroprotective therapy with PPI should be used for patients considered to be at increased risk from chronic aspirin therapy. Increased risk was defined as a history of ulcer disease or complication, dual antiplatelet therapy, concomitant anticoagulant therapy, or more than one of the following risk factors: age $\geq 60$ years, glucocorticoid use, or dyspepsia/gastroesophageal reflux disease (GERD). ${ }^{48}$ Recent concerns about interaction between PPI and clopidogrel is beyond the scope of this article, however it should be kept in mind when prescribing them together. ${ }^{49}$

In summary, further research is needed to determine the optimal regimen for primary prevention of GI toxicity among "high risk" low dose aspirin users. Based upon the currently available evidence, esomeprazole is very effective for secondary prevention of GI toxicity in low dose aspirin users.

\section{Disclosure}

The authors report no conflicts of interest in this work.

\section{References}

1. Collaborative overview of randomised trials of antiplatelet therapy. In: Prevention of death, myocardial infarction, and stroke by prolonged antiplatelet therapy in various categories of patients. Antiplatelet Trialists' Collaboration. BMJ. 1994;308(6921):81-106.

2. Weisman SM, Graham DY. Evaluation of the benefits and risks of low-dose aspirin in the secondary prevention of cardiovascular and cerebrovascular events. Arch Intern Med. 2002;162(19):2197-2202.

3. Yeomans N, Lanas A, Labenz J, et al. Efficacy of esomeprazole (20 mg once daily) for reducing the risk of gastroduodenal ulcers associated with continuous use of low-dose aspirin. Am J Gastroenterol. 2008;103(10):2465-2473.

4. Laine L. Review article: gastrointestinal bleeding with low-dose aspirin what's the risk? Aliment Pharmacol Ther. 2006 Sep 15;24(6):897-908.

5. Derry S, Loke YK. Risk of gastrointestinal haemorrhage with long term use of aspirin: meta-analysis. BMJ. 2000;321(7270):1183-1187.

6. Wolfe MM, Lichtenstein DR, Singh G. Gastrointestinal toxicity of nonsteroidal antiinflammatory drugs. N Engl J Med. 1999;340(24):1888-1899.

7. Lanas A, Perez-Aisa MA, Feu F, et al. A nationwide study of mortality associated with hospital admission due to severe gastrointestinal events and those associated with nonsteroidal antiinflammatory drug use. Am J Gastroenterol. 2005;100(8):1685-1693.

8. Nakayama M, Iwakiri R, Hara M, et al. Low-dose aspirin is a prominent cause of bleeding ulcers in patients who underwent emergency endoscopy. J Gastroenterol. 2009;44(9):912-918.

9. Vane JR. Inhibition of prostaglandin synthesis as a mechanism of action for aspirin-like drugs. Nat New Biol. 1971;231(25):232-235.

10. Robert A. Cytoprotection by prostaglandins. Gastroenterology. 1979;77(4 Pt 1):761-767.

11. Feldman M, Shewmake K, Cryer B. Time course inhibition of gastric and platelet COX activity by acetylsalicylic acid in humans. Am J Physiol Gastrointest Liver Physiol. 2000;279(5):G1113-11120.

12. McQuaid KR, Laine L. Systematic review and meta-analysis of adverse events of low-dose aspirin and clopidogrel in randomized controlled trials. Am J Med. 2006 Aug;119(8):624-638.

13. Sorensen HT, Mellemkjaer L, Blot WJ, et al. Risk of upper gastrointestinal bleeding associated with use of low-dose aspirin. Am J Gastroenterol. 2000;95(9):2218-2224.

14. Lanas A, Bajador E, Serrano P, et al. Nitrovasodilators, low-dose aspirin, other nonsteroidal antiinflammatory drugs, and the risk of upper gastrointestinal bleeding. N Engl J Med. 2000;343(12):834-839.

15. Serrano P, Lanas A, Arroyo MT, Ferreira IJ. Risk of upper gastrointestinal bleeding in patients taking low-dose aspirin for the prevention of cardiovascular diseases. Aliment Pharmacol Ther. 2002;16(11):1945-1953. 
16. Weil J, Colin-Jones D, Langman M, et al. Prophylactic aspirin and risk of peptic ulcer bleeding. BMJ. 1995;310(6983):827-830.

17. Huang JQ, Sridhar S, Hunt RH. Role of Helicobacter pylori infection and non-steroidal anti-inflammatory drugs in peptic-ulcer disease: a meta-analysis. Lancet. 2002;359(9300):14-22.

18. Chan FK, Chung SC, Suen BY, et al. Preventing recurrent upper gastrointestinal bleeding in patients with Helicobacter pylori infection who are taking low-dose aspirin or naproxen. $N$ Engl J Med. 2001;344(13):967-973.

19. Lai KC, Lam SK, Chu KM, et al. Lansoprazole for the prevention of recurrences of ulcer complications from long-term low-dose aspirin use. $N$ Engl J Med. 2002;346(26):2033-2038.

20. Chan FK. Long-term incidence of ulcer bleeding with low-dose aspiring after eradication of $\mathrm{H}$. pylori: A 4-year prospective cohort study [abstract]. Gastroenterology. 2005;128:A133.

21. Pilotto A, Franceschi M, Longoa MG, et al. Helicobacter pylori infection and the prevention of peptic ulcer with proton pump inhibitors in elderly subjects taking low-dose aspirin. Dig Liver Dis. 2004;36(10):666-670.

22. Yusuf S, Zhao F, Mehta SR, Chrolavicius S, Tognoni G, Fox KK. Effects of clopidogrel in addition to aspirin in patients with acute coronary syndromes without ST-segment elevation. $N$ Engl J Med. 2001;345(7):494-502.

23. Diener HC, Bogousslavsky J, Brass LM, et al. Aspirin and clopidogrel compared with clopidogrel alone after recent ischaemic stroke or transient ischaemic attack in high-risk patients (MATCH): randomised, doubleblind, placebo-controlled trial. Lancet. 2004;364(9431):331-337.

24. Bhatt DL, Fox KA, Hacke W, et al. Clopidogrel and aspirin versus aspirin alone for the prevention of atherothrombotic events. $N$ Engl J Med. 2006;354(16):1706-1717.

25. Hansson L, Zanchetti A, Carruthers SG, et al. Effects of intensive bloodpressure lowering and low-dose aspirin in patients with hypertension: principal results of the Hypertension Optimal Treatment (HOT) randomised trial. HOT Study Group. Lancet. 1998;351(9118):1755-1762.

26. Patrono C, Garcia Rodriguez LA, Landolfi R, Baigent C. Lowdose aspirin for the prevention of atherothrombosis. $N$ Engl $J$ Med. 2005;353(22):2373-2383.

27. Andreotti F, Testa L, Biondi-Zoccai GG, Crea F. Aspirin plus warfarin compared to aspirin alone after acute coronary syndromes: an updated and comprehensive meta-analysis of 25,307 patients. Eur Heart J. 2006;27(5):519-526.

28. Nielsen GL, Sorensen HT, Mellemkjoer L, et al. Risk of hospitalization resulting from upper gastrointestinal bleeding among patients taking corticosteroids: a register-based cohort study. Am J Med. 2001;111(7):541-545.

29. de Abajo FJ, Garcia Rodriguez LA. Risk of upper gastrointestinal bleeding and perforation associated with low-dose aspirin as plain and enteric-coated formulations. BMC Clin Pharmacol. 2001;1:1.

30. Taylor DW, Barnett HJ, Haynes RB, et al. Low-dose and high-dose acetylsalicylic acid for patients undergoing carotid endarterectomy: a randomised controlled trial. ASA and Carotid Endarterectomy (ACE) Trial Collaborators. Lancet. 1999;353(9171):2179-2184.

31. Baron JA, Cole BF, Sandler RS, et al. A randomized trial of aspirin to prevent colorectal adenomas. $N$ Engl J Med. 2003;348(10):891-899.

32. Kelly JP, Kaufman DW, Jurgelon JM, Sheehan J, Koff RS, Shapiro S. Risk of aspirin-associated major upper-gastrointestinal bleeding with entericcoated or buffered product. Lancet. 1996;348(9039):1413-1416.

33. Penney AG, Andrews FJ, O'Brien PE. Effects of misoprostol on delayed ulcer healing induced by aspirin. Dig Dis Sci. 1994;39(5):934-939.
34. Roth S, Agrawal N, Mahowald M, et al. Misoprostol heals gastroduodenal injury in patients with rheumatoid arthritis receiving aspirin. Arch Intern Med. 1989;149(4):775-779.

35. Watanabe T, Sugimori S, Kameda N, et al. Small bowel injury by low-dose enteric-coated aspirin and treatment with misoprostol: a pilot study. Clin Gastroenterol Hepatol. 2008;6(11):1279-1282.

36. Donnelly MT, Goddard AF, Filipowicz B, Morant SV, Shield MJ, Hawkey CJ. Low-dose misoprostol for the prevention of low-dose aspirin-induced gastroduodenal injury. Aliment Pharmacol Ther. 2000;14(5):529-534.

37. Rostom A, Dube C, Wells G, et al. Prevention of NSAID-induced gastroduodenal ulcers. Cochrane Database Syst Rev. 2002(4):CD002296.

38. Leontiadis GI, Sreedharan A, Dorward S, et al. Systematic reviews of the clinical effectiveness and cost-effectiveness of proton pump inhibitors in acute upper gastrointestinal bleeding. Health Technol Assess. 2007;11(51):iii-iv, 1-164.

39. Hawkey CJ, Karrasch JA, Szczepanski L, et al. Omeprazole compared with misoprostol for ulcers associated with nonsteroidal antiinflammatory drugs. Omeprazole versus Misoprostol for NSAID-induced Ulcer Management (OMNIUM) Study Group. $N$ Engl $J$ Med. 1998;338(11):727-734.

40. Yeomans ND, Tulassay Z, Juhasz L, et al. A comparison of omeprazole with ranitidine for ulcers associated with nonsteroidal antiinflammatory drugs. Acid Suppression Trial: Ranitidine versus Omeprazole for NSAID-associated Ulcer Treatment (ASTRONAUT) Study Group. N Engl J Med. 1998;338(11):719-726.

41. Lanas A, Rodrigo L, Marquez JL, et al. Low frequency of upper gastrointestinal complications in a cohort of high-risk patients taking low-dose aspirin or NSAIDS and omeprazole. Scand J Gastroenterol. 2003;38(7):693-700.

42. Goldstein JL, Johanson JF, Suchower LJ, Brown KA. Healing of gastric ulcers with esomeprazole versus ranitidine in patients who continued to receive NSAID therapy: a randomized trial. Am J Gastroenterol. 2005;100(12):2650-2657.

43. Scheiman JM, Yeomans ND, Talley NJ, et al. Prevention of ulcers by esomeprazole in at-risk patients using non-selective NSAIDs and COX-2 inhibitors. Am J Gastroenterol. 2006;101(4):701-710.

44. Chan FK, Ching JY, Hung LC, et al. Clopidogrel versus aspirin and esomeprazole to prevent recurrent ulcer bleeding. $N$ Engl $J$ Med. 2005;352(3):238-244.

45. Lai KC, Chu KM, Hui WM, et al. Esomeprazole with aspirin versus clopidogrel for prevention of recurrent gastrointestinal ulcer complications. Clin Gastroenterol Hepatol. 2006;4(7):860-865.

46. Vachhani R, Olds G, Velanovich V. Esomeprazole: a proton pump inhibitor. Expert Rev Gastroenterol Hepatol. 2009;3(1):15-27.

47. Niazi M, Andersson T, Naucler E, Sundin M, Naesdal J. Evaluation of the pharmacokinetic interaction between esomeprazole $(40 \mathrm{mg})$ and acetylsalicylic acid $(325 \mathrm{mg})$ in healthy volunteers. Int J Clin Pharmacol Ther. 2009;47(9):564-569.

48. Bhatt DL, Scheiman J, Abraham NS, et al. ACCF/ACG/AHA 2008 expert consensus document on reducing the gastrointestinal risks of antiplatelet therapy and NSAID use. Am J Gastroenterol. 2008;103(11):2890-2907.

49. Laine L, Hennekens C. Proton pump inhibitor and clopidogrel interaction: fact or fiction? Am J Gastroenterol. 2010;105(1):34-41.
Therapeutics and Clinical Risk Management

\section{Publish your work in this journal}

Therapeutics and Clinical Risk Management is an international, peerreviewed journal of clinical therapeutics and risk management, focusing on concise rapid reporting of clinical studies in all therapeutic areas, outcomes, safety, and programs for the effective, safe, and sustained use of medicines. This journal is indexed on PubMed Central, CAS,

\section{Dovepress}

EMBase, Scopus and the Elsevier Bibliographic databases. The manuscript management system is completely online and includes a very quick and fair peer-review system, which is all easy to use. Visit http://www.dovepress.com/testimonials.php to read real quotes from published authors. 